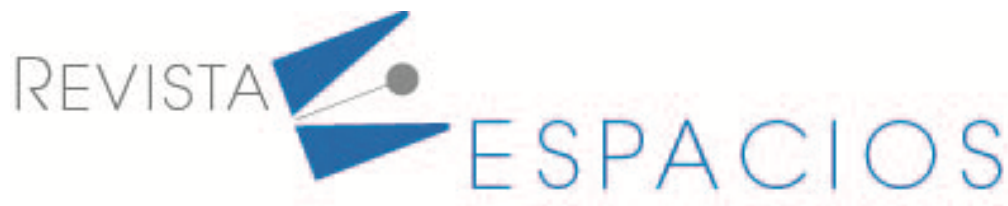

\title{
Propiedades psicométricas de un cuestionario para medir la calidad en educación física
}

\author{
Psychometric properties of a questionnaire to measure quality in physical education in \\ English
}

DEL VAL MARTÍN, Pablo ${ }^{1}$

\begin{abstract}
Resumen
El objetivo fue analizar los parámetros psicométricos y las estructuras factoriales del Cuestionario de EFC en el contexto ecuatoriano. La muestra estuvo conformada por 374 profesionales de Educación Física. Se utilizó el cuestionario Índice Global de la Educación Física de Calidad. Se realizó un Análisis Factorial Exploratorio (AFE) para evaluar la estructura factorial y el análisis se implementó utilizando una matriz policórica y el método de extracción Robust Diagonally Weighted Least Squares (RDWLS). Los tests de esfericidad de Bartlett $(4092,8, g l=1225, p<.001)$ e KMO (.97) sugieren la interpretabilidad muy buena de la matriz de correlación de los ítems.

Palabras clave: Políticas Educativas, desarrollo, escala, validación.
\end{abstract}

\begin{abstract}
The objective was to analyze the psychometric parameters and factorial structures of the OBE Questionnaire in the Ecuadorian context. The sample consisted of 374 Physical Education professionals. The Global Quality Physical Education Index questionnaire was used. An Exploratory Factor Analysis (EFA) was performed to evaluate the factor structure and the analysis was implemented using a polychoric matrix and the Robust Diagonally Weighted Least Squares (RDWLS) extraction method. The Bartlett sphericity tests $(4092.8, \mathrm{gl}=1225, \mathrm{p}<.001)$ and $\mathrm{KMO}(.97)$ suggest the very good interpretability of the correlation matrix of the items.

Key words: Educational policies, development, scale, validation.
\end{abstract}

\section{Introducción}

Hoy en día nadie duda de la importancia que tiene la Educación Física (EF en adelante) en la formación de nuestros alumnos, debido a que "la asignatura de Educación Física puede ser el eje vertebrador de las alternativas de ocio saludables que aumenten la actividad física del alumnado" (Vera et al., 2018, p. 665). Es de sobra conocido que a través de la EF se pueden generar múltiples beneficios en la salud; desde los que quizás son más obvios y han sido más estudiados y que se vinculan a beneficios estrictamente físicos, hasta los que resultan más novedosos y emergentes y que gracias a la neuroeducación podemos comprender cada día más, como los beneficios a nivel de salud emocional o social (Pellicer, 2015). 
La finalidad de la EF (como área curricular específica dentro de un sistema educativo universal, obligatorio y público), es el desarrollo físicomotriz del alumnado, la promoción de la cultura física y su aportación al planteamiento global de desarrollo integral, como ciudadanos de una sociedad democrática, fundamentalmente en el desarrollo de las competencias clave y en el desarrollo integral del alumnado desde, con y a través de la motricidad (López et al., 2016), desarrollo que se fomenta en los centros educativos, dado que éstos posibilitan la transmisión de valores, actitudes y normas de ciudadanía al alumnado (Contreras et al., 2010). La experiencia de aprendizaje que se ofrece a los niños y jóvenes a través de las clases de EF debe ser apropiada para ayudarles a adquirir las habilidades psicomotrices, la comprensión cognitiva y las aptitudes sociales y emocionales que necesitan para llevar una vida físicamente activa (UNESCO, 2015), de ahí la importancia de que esas experiencias de aprendizaje sean de calidad.

El concepto de Educación Física de calidad (EFC en adelante) -QPE por sus siglas en inglés- fue planteado por la UNESCO en el año 2005, definiéndola como "la experiencia de aprendizaje planificada, progresiva e inclusiva que forma parte del currículo en educación infantil, primaria y secundaria” (UNESCO, 2005, p.9). Esta definición nos deja entrever las bases principales a tener en cuenta para alcanzar una EF de calidad: el aprendizaje y la planificación progresiva. Más tarde, en el 2015, la propia UNESCO publicó una guía destinada a los responsables políticos de los Estados miembros de la UNESCO con el objetivo de ayudar en la formulación e implementación de programas y políticas de EFC inclusivos (UNESCO, 2015).

Esta iniciativa nos demuestra lo importante y necesaria que es la EF y el hecho de que ésta sea de calidad. De hecho, son muchos los estudios que han surgido desde entonces enfocados en medir la calidad de la EF (del Val et al., 2021; Ho et al., 2021; Rodríguez et al., 2021; Ho et al., 2019; Lara et al., 2018).

\subsection{La Educación Física en Ecuador}

En Ecuador a partir del 2014 comenzó a gestarse a raíz del acuerdo ministerial 0041-14, el nuevo currículo de la Educación General Básica, mismo que fue aprobado en el 2016 y que como principal novedad para el área de EF contempló el aumento de horas de clase, pasando éstas de 2 a 5 horas de clase semanales.

A pesar de que uno de los principales argumentos para este aumento de horas responda al aumento de los índices de obesidad infantil y juvenil (ENSANUT, 2014), no podemos negar que se trata de una situación idílica para el área de EF, pues además de los obvios beneficios que la EF genera sobre la salud del alumnado, es de sobra conocido que proporciona otra serie de beneficios, como los afectivomotivacionales, los de inserción social, los cognitivos o los de relaciones interpersonales (Pérez Pueyo et al.,2020).

Sin embargo, y a pesar de contar con un nuevo currículo que dispone más horas y contenidos actualizados para EF, se han observado debilidades en la práctica docente de los profesionales del área, donde persiste la aplicación de modelos pedagógicos tradicionales (Villafuerte et al., 2019). Lógicamente, y a pesar de que la valoración de la EF por parte de los alumnos es positiva, así como el aumento de horas de ésta (del Val et al., 2021), estas prácticas han impactado en los estudiantes, quienes se reúsan a la práctica deportiva a pesar de su potencial contribución a la salud y bienestar emocional de las personas (López Pastor et al., 2016).

(Ho et al., 2021) señalan que son numerosas las cuestiones a tener en cuenta para alcanzar una EF de calidad, como, por ejemplo, el debate sobre la obligatoriedad de ésta para todos los niños sin discriminación, una asignación de tiempo inadecuada, los problemas de personal (falta del mismo o insuficiente capacitación), la falta de apoyo gubernamental o la necesidad de reconocimiento oficial del tema. No obstante, por encima de todas estas cuestiones destaca la falta de una herramienta de medición de la mencionada calidad en EF, y es por eso por lo que el objetivo planteado para este estudio es examinar las propiedades psicométricas y estructura factorial del Cuestionario de Educación Física de Calidad. 


\section{Metodología}

\subsection{Tipo de estudio y participantes}

El presente estudio tiene un enfoque cuantitativo de carácter descriptivo y correlacional. La muestra estuvo conformada por 374 profesionales de EF de diferentes ciudades del Ecuador que fueron invitados a participar en este estudio; donde el $57.75 \%$ son hombres y $42,24 \%$ mujeres.El puesto de trabajo de los encuestados durante el período de investigación fue el siguiente: 139 (37,2\%) trabajaban como 'profesores de educación física en escuelas primarias', $125(33,4 \%)$ trabajaban como 'profesores de educación física en escuelas secundarias' y 110 $(29,4 \%)$ - el grupo 'otros' - trabajaban como profesores, profesores, conferencistas, entrenadores en universidades o en el gobierno, y en oficinas deportivas.

\subsection{Instrumento}

Se utilizó encuesta sobre el Índice Global de la Educación Física de Calidad (GIQPE, por sus siglas en inglés) de Ho et al. (2018) utilizada en estudios previos con poblaciones de docentes latinoamericanos y asiáticos, obteniendo adecuados valores de fiabilidad (á > .85) e índices de bondad y ajuste aceptables (Ho et al., 2019).

La encuesta está compuesta de 50 ítems en torno a la EFC, agrupados en ocho factores: desarrollo de habilidades y conciencia del cuerpo en las actividades físicas y deportivas; lugar, instalaciones y equipo de educación física; enseñanza de la educación física; planes para la factibilidad y accesibilidad de la educación física para todos los estudiantes; normas sociales y práctica cultural; aportación gubernamental para el mejoramiento de la calidad de la educación física; habilidad cognitiva, pensamiento independiente y otras habilidades de desarrollo genérico; desarrollo de programas alternativos en educación física.

El instrumento está dividido en dos partes, en la primera se detallan los datos sociodemográficos (ciudad, género, institución, años de experiencia laboral, nivel donde trabaja y tipo de escuela), y en la segunda, las preguntas sobre la percepción de la EFC en su entorno/ciudad, con una escala de respuesta donde 1 = no alcanzado totalmente y 10 = completamente alcanzado.

\subsection{Procedimiento}

El instrumento original en inglés fue traducido al castellano hablado en Latinoamérica siguiendo el procedimiento backtranslation (Hambleton y Kanjee, 1995). La encuesta se administró a través de Google Forms solicitando la colaboración de profesionales vinculados a la EF de todo el país, garantizando su anonimato y agradeciendo de antemano el tiempo dedicado.

\subsection{Análisis de datos}

Se realizó un Análisis Factorial Exploratorio (AFE) para evaluar la estructura factorial para el Índice Global de la Educación Física de Calidad (GIQPE) (Ho, Ahmed y Kukurova, 2021). El análisis se implementó utilizando una matriz policórica y el método de extracción Robust Diagonally Weighted Least Squares (RDWLS) (Asparouhov y Muthen, 2010). La decisión sobre el número de factores a retener se tomó mediante la técnica de Análisis Paralelo con permutación aleatoria de los datos observados (Timmerman, y Lorenzo-Seva, 2011) y la rotación utilizada fue Robust Promin (Lorenzo-Seva y Ferrando, 2019).

La idoneidad del modelo se evaluó mediante los índices de ajuste Root Mean Square Error of Aproximation (RMSEA), Comparative Fit Index (CFI) y Tucker-Lewis Index (TLI). Según la literatura (Brown, 2006), los valores de RMSEA deben ser inferiores a 0,08 , con un intervalo de confianza que no llegue a 0,10 , y los valores de CFI y TLI deben estar por encima de 0,90, o preferiblemente, 0,95. 
La estabilidad de los factores se evaluó mediante el índice $H$ (Ferrando y Lorenzo-Seva, 2018). El índice $H$ evalúa qué tan bien un conjunto de ítems representa un factor común (Ferrando y Lorenzo-Seva, 2018). Los valores de $H$ oscilan entre 0 y 1.

Los valores altos de $H(>0,80)$ sugieren una variable latente bien definida, que es más probable que sea estable en diferentes estudios. Valores de $H$ bajos sugieren una variable latente mal definida y probablemente inestable entre diferentes estudios (Ferrando y Lorenzo-Seva, 2018). Finalmente, el parámetro de discriminación del ítem y los umbrales fueron evaluados usando la parametrización Reckase (Reckase, 1985). El AFE fue hecho en el programa Factor Analysis (Lorenzo-Seva y Ferrando, 2006).

\section{Resultados y discusión}

Los tests de esfericidad de Bartlett $(4092,8, g l=1225, p<.001)$ e KMO (.97) sugieren la interpretabilidad muy buena de la matriz de correlación de los ítems. La Análisis Paralela sugirió dos factores como los mas representativos para los datos (Tabla 1 ).

Tabla 1

Resultados de la Análisis Paralela

\begin{tabular}{|c|c|c|}
\hline Factores & $\begin{array}{l}\text { Porcentual de variancia explicada } \\
\text { de los datos reales }\end{array}$ & $\begin{array}{l}\text { Porcentual de variancia explicada de } \\
\text { los datos aleatorios ( } 95 \% \text { IC) }\end{array}$ \\
\hline 1 & $58.8430 *$ & 4.5775 \\
\hline 2 & 6.9869* & 4.3058 \\
\hline 3 & 3.7579 & 4.1243 \\
\hline 4 & 2.7985 & 3.9637 \\
\hline 5 & 2.1434 & 3.8336 \\
\hline 6 & 1.6674 & 3.6881 \\
\hline 7 & 1.5859 & 3.5771 \\
\hline 8 & 1.3436 & 3.4766 \\
\hline 9 & 1.3017 & 3.3746 \\
\hline 10 & 1.1542 & 3.2650 \\
\hline 11 & 1.1185 & 3.1551 \\
\hline 12 & 1.0400 & 3.0773 \\
\hline 13 & .9638 & 2.9872 \\
\hline 14 & .9120 & 2.9019 \\
\hline 15 & .9015 & 2.8209 \\
\hline 16 & .8348 & 2.7286 \\
\hline 17 & .7911 & 2.6589 \\
\hline 18 & .7723 & 2.5755 \\
\hline 19 & .7596 & 2.5020 \\
\hline 20 & .7162 & 2.4251 \\
\hline 21 & .6695 & 2.3425 \\
\hline 22 & .6460 & 2.2696 \\
\hline 23 & .6286 & 2.2041 \\
\hline 24 & .5958 & 2.1289 \\
\hline 25 & .5676 & 2.0654 \\
\hline 26 & .5609 & 1.9894 \\
\hline 27 & .5395 & 1.9297 \\
\hline 28 & .5043 & 1.8545 \\
\hline 29 & .4959 & 1.7911 \\
\hline 30 & .4384 & 1.7181 \\
\hline
\end{tabular}




\begin{tabular}{|c|c|c|}
\hline Factores & $\begin{array}{l}\text { Porcentual de variancia explicada } \\
\text { de los datos reales }\end{array}$ & $\begin{array}{l}\text { Porcentual de variancia explicada de } \\
\text { los datos aleatorios ( } 95 \% \text { IC) }\end{array}$ \\
\hline 31 & .3973 & 1.6505 \\
\hline 32 & .3789 & 1.5779 \\
\hline 33 & .3535 & 1.5135 \\
\hline 34 & .3288 & 1.4344 \\
\hline 35 & .3041 & 1.3719 \\
\hline 36 & .2836 & 1.3139 \\
\hline 37 & .2637 & 1.2344 \\
\hline 38 & .2608 & 1.1812 \\
\hline 39 & .2392 & 1.0967 \\
\hline 40 & .2127 & 1.0282 \\
\hline 41 & .1979 & .9579 \\
\hline 42 & .1926 & .8835 \\
\hline 43 & .1786 & .7926 \\
\hline 44 & .1293 & .7095 \\
\hline 45 & .1020 & .6271 \\
\hline 46 & .0650 & .5425 \\
\hline 47 & .0465 & .4578 \\
\hline 48 & .0237 & .3576 \\
\hline 49 & .0028 & .2471 \\
\hline
\end{tabular}

Nota: El número de factores que se deben retener es dos, ya que dos factores de los datos reales tienen un \% de varianza explicada mayor que los datos aleatorios.

Los ítems presentaron cargas factoriales adecuadas, con cargas factoriales elevadas en sus respectivos factores (en negrito; Tabla 3). Solo dos ítems tenían un patrón de cargas cruzadas, es decir, ítems con cargas factoriales superiores a 0.30 en más de un factor.

Los índices de ajuste del instrumento fueron excelentes $\left(x^{2}=628,63, \mathrm{gl}=853 ; p=0,999 ; \mathrm{RMSEA}=1,000\right.$; $\mathrm{CFI}=1,000 ; \mathrm{TLI}=1,000$ ). La fiabilidad compuesta de los factores también resultó aceptable (por encima de $0,70)$ para casi todos los factores, excepto para el factor 3 "Quality Teaching of Physical Education" (CC = 0,091).

La medida de replicabilidad de la estructura factorial (índice H, Ferrando y Lorenzo-Seva, 2018) sugirió que el factor 3 puede ser replicable en estudios futuros $(H>0,80)$, ya que la confiabilidad compuesta también está asociado al número de los ítems $>0,30$.

\section{Tabla 2}

ítems del Índice Global de la Educación

\begin{tabular}{|c|c|}
\hline No & Descripción del ítem \\
\hline 1. & $\begin{array}{l}\text { La autoridad educativa de nuestra ciudad tiene una clara relación con instituciones financieras internacionales para asegurar } \\
\text { que la educación física esté incluida como parte de sus programas de ayuda en educación. }\end{array}$ \\
\hline 2. & $\begin{array}{l}\text { El programa de educación física de nuestra ciudad anima a los estudiantes a aprender e interactuar con los compañeros de } \\
\text { clase. }\end{array}$ \\
\hline 3. & $\begin{array}{l}\text { Las escuelas en nuestra ciudad tienen instalaciones seguras y apropiadas para la enseñanza y el aprendizaje de la educación } \\
\text { física. }\end{array}$ \\
\hline 4. & $\begin{array}{l}\text { Nuestro programa de educación física contiene elementos que ayudan a desarrollar las destrezas básicas de los estudiantes } \\
\text { para distintas actividades físicas y deportivas. }\end{array}$ \\
\hline 5. & $\begin{array}{l}\text { Las escuelas en nuestra ciudad tienen un ambiente seguro y apropiado para la enseñanza y el aprendizaje de la educación } \\
\text { física. }\end{array}$ \\
\hline 6. & $\begin{array}{l}\text { Las actitudes positivas relacionadas con el deporte y los valores son el centro del aprendizaje en la educación física, en } \\
\text { nuestra ciudad. }\end{array}$ \\
\hline
\end{tabular}




\begin{tabular}{|c|c|}
\hline No & Descripción del ítem \\
\hline 7. & $\begin{array}{l}\text { El programa de educación física contiene elementos de enseñanza que ayudan a los estudiantes a desarrollar su } \\
\text { comprensión básica de la importancia de actividades física y salud. }\end{array}$ \\
\hline 8. & $\begin{array}{l}\text { La autoridad educativa de la ciudad tiene una política clara de incentivar el desarrollo oportunidades de igualdad de } \\
\text { aprendizaje en el programa de educación física de la escuela. }\end{array}$ \\
\hline 9. & $\begin{array}{l}\text { El programa de educación física de nuestra ciudad incentiva a los estudiantes a asumir responsabilidades apropiadas para } \\
\text { servir en clubes deportivos u otras actividades similares en la escuela o en la comunidad. }\end{array}$ \\
\hline 10. & $\begin{array}{l}\text { Nuestra ciudad tiene una política adecuada para reforzar la accesibilidad de la enseñanza de la educación física a todos los } \\
\text { niños, independientemente de su capacidad/ discapacidad, sexo, edad, cultura, raza/ etnicidad, religión y origen social o } \\
\text { económico. }\end{array}$ \\
\hline 11. & $\begin{array}{l}\text { El programa de educación física de nuestra ciudad contiene elementos que ayudan a desarrollar en los estudiantes su } \\
\text { comprensión adecuada de la salud y la aptitud física, incluyendo el establecimiento y logro de metas personales para una } \\
\text { vida sana. }\end{array}$ \\
\hline 12. & $\begin{array}{l}\text { El programa de educación física en las escuelas en nuestra ciudad ayuda a los estudiantes con el desarrollo de sus } \\
\text { habilidades del pensamiento crítico. }\end{array}$ \\
\hline 13. & $\begin{array}{l}\text { Nuestra ciudad tiene una política apropiada extendiendo las oportunidades para el aprendizaje en actividades físicas a través } \\
\text { de programas relacionados con actividades deportivas, programas de actividades después de clases, programas de } \\
\text { actividades extra-curriculares en las escuelas. }\end{array}$ \\
\hline 14. & $\begin{array}{l}\text { El programa de educación física de nuestra ciudad contiene oportunidades para acciones apropiadas para ayudar a los } \\
\text { estudiantes a mantener estilos de vida activos y saludables. }\end{array}$ \\
\hline 15. & $\begin{array}{l}\text { Nuestra autoridad educativa tiene un claro reconocimiento del rol característico de la educación física como parte de un } \\
\text { sistema educativo balanceado para la realización del potencial humano, la salud y el bienestar de todos los ciudadanos. }\end{array}$ \\
\hline 16. & El programa de educación física de nuestra ciudad ayuda a los estudiantes a desarrollar hábitos de ejercicio. \\
\hline 17. & Los hábitos de educación física y deporte están bien desarrollados de acuerdo al programa de educación física de la escuela. \\
\hline 18. & $\begin{array}{l}\text { Distintos tipos de actividad física y conocimientos asociados son los contenidos de aprendizaje de educación física en nuestra } \\
\text { ciudad. }\end{array}$ \\
\hline 19. & $\begin{array}{l}\text { La autoridad en educación de la ciudad tiene planes de incentivar a cada estudiante a participar en deportes y actividades } \\
\text { físicas luego de su horario escolar y a que use su tiempo libre sabiamente en deporte y actividades físicas. }\end{array}$ \\
\hline 20. & $\begin{array}{l}\text { Nuestra autoridad en educación tiene una política clara en cuanto al apoyo de investigaciones para mejorar la efectividad y } \\
\text { calidad de los programas de educación física en las escuelas }\end{array}$ \\
\hline 21. & $\begin{array}{l}\text { El conocimiento en salud es considerado una de las áreas más importantes para aprender sobre la actividad física en nuestra } \\
\text { ciudad. }\end{array}$ \\
\hline 22. & $\begin{array}{l}\text { El programa de educación física en nuestra ciudad ayuda al estudiante a desarrollarse competentemente en diferentes } \\
\text { actividades físicas y deportivas. }\end{array}$ \\
\hline 23. & $\begin{array}{l}\text { Nuestro programa de educación física provee a estudiantes con oportunidades para que tengan parte en diferentes } \\
\text { actividades físicas. }\end{array}$ \\
\hline 24. & $\begin{array}{l}\text { Nuestro programa de educación física ayuda al estudiante a entender la relación entre actividades físicas y deportivas y } \\
\text { desarrollo personal y social. }\end{array}$ \\
\hline 25. & $\begin{array}{l}\text { Nuestro programa de educación física contiene elementos de enseñanza que permiten ayudar a estudiantes a comunicar } \\
\text { afectivamente sus ideas y sentimientos con otros. }\end{array}$ \\
\hline 26. & $\begin{array}{l}\text { Las escuelas en nuestra ciudad tienen un equipamiento acorde y seguro para la enseñanza y aprendizaje de la educación } \\
\text { física }\end{array}$ \\
\hline 27. & $\begin{array}{l}\text { El conocimiento acerca del funcionamiento de nuestros cuerpos y su relación con las actividades están siendo bien } \\
\text { enseñadas a través del programa de educación física de la escuela. }\end{array}$ \\
\hline 28. & $\begin{array}{l}\text { La autoridad de educación en nuestra ciudad reconoce que la educación física de buena calidad depende de educadores } \\
\text { cualificados, por consiguiente, la prioridad es para el entrenamiento del personal calificados, aun cuando otras dependencias } \\
\text { estén cortas en suministros. }\end{array}$ \\
\hline 29. & La educación física es una materia obligatoria para los estudiantes de bachillerato en sus últimos años en nuestra ciudad. \\
\hline 30. & $\begin{array}{l}\text { El programa de educación física de las escuelas en nuestra ciudad cultiva la habilidad del pensamiento independiente en el } \\
\text { estudiante. }\end{array}$ \\
\hline 31. & La educación física es una materia obligatoria para los estudiantes de bachillerato en nuestra ciudad. \\
\hline 32. & $\begin{array}{l}\text { El conocimiento de actividades físicas y deporte están siendo bien enseñadas a través del programa de educación física de la } \\
\text { escuela. }\end{array}$ \\
\hline
\end{tabular}




\begin{tabular}{|c|c|}
\hline No & Descripción del ítem \\
\hline 33. & $\begin{array}{l}\text { Los programas de educación física en las escuelas de nuestra ciudad realzan el desarrollo de la habilidad de los estudiantes } \\
\text { para resolver problemas. }\end{array}$ \\
\hline 34. & $\begin{array}{l}\text { A los estudiantes en nuestra ciudad se les dan oportunidades adecuadas para aprender activamente durante las lecciones de } \\
\text { actividad física. }\end{array}$ \\
\hline 35. & $\begin{array}{l}\text { La autoridad de educación de la ciudad tiene una política clara para lograr el desarrollo de que ambos géneros tengan } \\
\text { oportunidades iguales para aprender en el programa de educación física de la escuela. }\end{array}$ \\
\hline 36. & $\begin{array}{l}\text { El conocimiento del crecimiento y su relación con actividades están bien impartidas a través del programa escolar de } \\
\text { educación física. }\end{array}$ \\
\hline 37. & $\begin{array}{l}\text { El programa de educación física en nuestra ciudad ayuda al estudiante a desarrollar las habilidades necesarias para participar } \\
\text { en actividades, dentro y fuera de la escuela, que tengan el potencial para generar participación e involucramiento a lo largo } \\
\text { de la vida, disponibles en la comunidad. }\end{array}$ \\
\hline 38. & $\begin{array}{l}\text { El programa de educación física en las escuelas de nuestra ciudad realza la habilidad del pensamiento innovador del } \\
\text { estudiante. }\end{array}$ \\
\hline 39. & $\begin{array}{l}\text { El programa de educación física en las escuelas de nuestra ciudad ayuda al estudiante a desarrollar el pensamiento moral y } \\
\text { conducta socialmente aceptable. }\end{array}$ \\
\hline 40 & $\begin{array}{l}\text { La autoridad de educación en nuestra ciudad promueve planes de colaboración internacionales frecuentemente entre } \\
\text { institutos para la preparación acerca de la calidad del desarrollo de la educación física en las escuelas }\end{array}$ \\
\hline 41. & $\begin{array}{l}\text { El programa de educación física en nuestra ciudad contiene elementos de enseñanza que ayudan a los estudiantes a } \\
\text { desarrollar sus habilidades motoras básicas dentro del contexto de actividades físicas apropiadas. }\end{array}$ \\
\hline 42. & $\begin{array}{l}\text { El programa de educación física en nuestra ciudad es el medio más efectivo para equipar a los niños con habilidades, } \\
\text { actitudes, valores, conocimiento, entendimiento y participación en actividad física y deporte a lo largo de la vida. }\end{array}$ \\
\hline 43. & $\begin{array}{l}\text { La autoridad en educación de nuestra ciudad tiene una política clara de solventar los problemas económicos en el desarrollo } \\
\text { de oportunidades de aprendizaje igualitario en el programa de educación física de la escuela. }\end{array}$ \\
\hline 44. & Todos los profesores de educación física en nuestra ciudad están calificados para enseñar educación física. \\
\hline 45. & La educación física es una materia obligatoria para todos los estudiantes de primaria en nuestra ciudad. \\
\hline 46. & $\begin{array}{l}\text { La autoridad en educación de nuestra ciudad ha implementado políticas para la educación física como un elemento de } \\
\text { derechos humanos para todos los niños. }\end{array}$ \\
\hline 47. & $\begin{array}{l}\text { El desarrollo de las habilidades de los estudiantes está siendo efectivamente realzadas a través del programa de educación } \\
\text { física de la escuela. }\end{array}$ \\
\hline 48. & $\begin{array}{l}\text { La autoridad en educación de nuestra ciudad fomenta la colaboración de institutos entre ciudades acerca de planes en } \\
\text { preparación del desarrollo de la calidad de la educación física en las escuelas. }\end{array}$ \\
\hline 49. & $\begin{array}{l}\text { El programa de educación física de nuestra ciudad contiene elementos de enseñanza que ayuda al estudiante a desarrollar } \\
\text { sus habilidades básicas en cuanto a la toma de decisiones y comunicación. }\end{array}$ \\
\hline 50. & La enseñanza y el aprendizaje de la educación física en nuestras escuelas es divertida y agradable. \\
\hline
\end{tabular}

Tabla 3

Estructura Factorial del Índice Global de

la Educación Física de Calidad (GIQPE)

\begin{tabular}{|c|c|c|c|c|c|c|c|c|}
\hline Ítems & Factor 1 & Factor 2 & Factor 3 & Factor 4 & Factor 5 & Factor 6 & Factor 7 & Factor 8 \\
\hline $\mathrm{V} 1$ & .028 & -.315 & .150 & .012 & 0.269 & -.098 & .736 & .025 \\
\hline $\mathrm{V} 2$ & .080 & .177 & .007 & -.011 & $\mathbf{0 . 6 2 5}$ & .053 & -.086 & -.044 \\
\hline $\mathrm{V} 3$ & .075 & -.259 & .027 & $\mathbf{1 . 0 4 1}$ & 0.026 & -.041 & $\mathbf{1 . 7 3 2}$ & .114 \\
\hline $\mathrm{V} 4$ & -.057 & .023 & .055 & .231 & $\mathbf{0 . 3 3 0}$ & .696 & -.090 & -.022 \\
\hline $\mathrm{V} 5$ & .075 & -.018 & .007 & $\mathbf{1 . 1 1 2}$ & 0.093 & -.041 & $\mathbf{1 . 5 0 9}$ & -.039 \\
\hline $\mathrm{V} 6$ & -.064 & .175 & .051 & .208 & $\mathbf{0 . 5 4 0}$ & .127 & .072 & -.051 \\
\hline $\mathrm{V} 7$ & -.040 & .132 & .079 & .202 & $\mathbf{0 . 5 1 3}$ &. $\mathbf{3 6 9}$ & -.089 & -.002 \\
\hline $\mathrm{V} 8$ & .359 & -.337 & .026 & .005 & $\mathbf{0 . 4 4 4}$ & -.054 & .632 & .059 \\
\hline $\mathrm{V} 9$ & -.140 & -.011 & -.065 & .029 & $\mathbf{0 . 6 0 0}$ & -.023 & .495 & .012 \\
\hline $\mathrm{V} 10$ & .283 & -.069 & -.096 & .012 & $\mathbf{0 . 4 9 7}$ & -.127 &. $\mathbf{4 9 8}$ & .045 \\
\hline $\mathrm{V} \mathrm{11}$ & .067 & .137 & -.016 & -.060 & $\mathbf{0 . 6 8 8}$ & .071 & -.004 & -.036 \\
\hline $\mathrm{V} 12$ & -.177 & .587 & .021 & -.046 & $\mathbf{0 . 5 0 6}$ & -.153 & -.024 & -.021 \\
\hline
\end{tabular}




\begin{tabular}{|c|c|c|c|c|c|c|c|c|}
\hline Ítems & Factor 1 & Factor 2 & Factor 3 & Factor 4 & Factor 5 & Factor 6 & Factor 7 & Factor 8 \\
\hline V 13 & -.056 & .109 & -.273 & .034 & 0.025 & .166 & .732 & .011 \\
\hline V 14 & -.014 & .167 & -.187 & .017 & 0.165 & .299 & .312 & -.005 \\
\hline V 15 & .182 & -.008 & -.219 & -.031 & 0.181 & .102 & .581 & .050 \\
\hline V 16 & -.035 & .289 & -.193 & -.052 & 0.247 & .413 & -.043 & -.056 \\
\hline V 17 & .061 & .050 & .138 & -.075 & 0.226 & .614 & -.089 & -.057 \\
\hline V 18 & .088 & .215 & -.092 & .011 & 0.295 & .285 & .028 & -.055 \\
\hline V 19 & .016 & -.152 & -.131 & -.015 & 0.043 & .214 & .861 & .028 \\
\hline V 20 & -.110 & .095 & -.058 & -.034 & 0.063 & -.072 & .813 & .017 \\
\hline V 21 & -.087 & .303 & -.147 & .024 & 0.006 & .258 & .464 & -.004 \\
\hline V 22 & -.041 & .409 & -.209 & -.032 & -0.016 & .539 & .091 & -.031 \\
\hline V 23 & -.001 & .152 & -.076 & .044 & -0.166 & .843 & .155 & -.020 \\
\hline V 24 & -.029 & .261 & .072 & -.131 & 0.008 & .720 & -.079 & .120 \\
\hline V 25 & -.166 & .712 & .088 & -.109 & 0.149 & .219 & -.089 & .145 \\
\hline V 26 & -.085 & .100 & .042 & .399 & -0.138 & .104 & 1.132 & -.020 \\
\hline V 27 & -.122 & .550 & .270 & .126 & 0.126 & .184 & .147 & .007 \\
\hline V 28 & -.117 & .378 & -.025 & .026 & -0.068 & -.058 & .678 & -.022 \\
\hline V 29 & .681 & .237 & -.127 & .128 & -0.064 & .021 & .065 & .852 \\
\hline V 30 & -.013 & .804 & .101 & -.051 & 0.125 & -.113 & .092 & .105 \\
\hline V 31 & .931 & -.076 & .036 & -.096 & 0.073 & -.113 & -.051 & .959 \\
\hline V 32 & -.022 & .628 & .301 & .003 & -0.033 & .171 & .030 & .043 \\
\hline V 33 & .010 & .990 & .057 & -.056 & 0.019 & -.118 & -.046 & -.037 \\
\hline V 34 & .248 & .838 & -.007 & .059 & 0.076 & -.120 & -.054 & .025 \\
\hline V 35 & .557 & .304 & .010 & -.018 & 0.170 & -.090 & .024 & .016 \\
\hline V 36 & .133 & .780 & .115 & .104 & 0.040 & -.006 & .069 & .021 \\
\hline V 37 & -.020 & .853 & -.102 & .002 & 0.052 & -.090 & .076 & .015 \\
\hline V 38 & -.114 & 1.255 & -.079 & -.035 & -0.029 & -.256 & .045 & .027 \\
\hline V 39 & -.006 & .978 & -.040 & -.040 & -0.101 & .014 & -.068 & .019 \\
\hline V 40 & -.088 & .219 & .141 & -.035 & -0.152 & -.126 & .819 & -.137 \\
\hline V 41 & .169 & .854 & -.104 & .061 & -0.087 & .083 & -.123 & .023 \\
\hline V 42 & .070 & 1.045 & -.069 & .046 & -.071 & -.052 & -.062 & -.067 \\
\hline V 43 & -.031 & .179 & .165 & .020 & .026 & -.183 & .731 & -.092 \\
\hline V 44 & .007 & .441 & .214 & -.011 & -.011 & -.098 & .239 & .017 \\
\hline V 45 & .779 & -.029 & -.064 & .028 & -.188 & .137 & -.019 & .498 \\
\hline V 46 & .565 & .053 & .083 & -.142 & .033 & -.044 & .185 & .007 \\
\hline V 47 & .151 & .314 & .192 & -.035 & -.099 & .391 & .012 & -.016 \\
\hline V 48 & .093 & -.012 & .128 & -.014 & -.131 & .031 & .753 & -.090 \\
\hline V 49 & .074 & .953 & -.002 & -.065 & -.058 & .029 & -.117 & -.062 \\
\hline V 50 & .187 & .667 & .086 & .146 & -.006 & .216 & -.118 & .006 \\
\hline Conf. compuesta & .819 & .957 & .091 & .926 & .778 & .803 & .977 & .828 \\
\hline H-latente & .894 & .990 & .804 & .939 & .939 & .974 & .971 & .894 \\
\hline H-observado & 1.081 & 1.348 & .841 & 1.009 & 1.221 & 1.273 & 1.236 & .901 \\
\hline
\end{tabular}

La relación de sensibilidad (SR) se puede interpretar como el número de niveles de factores diferentes que se pueden diferenciar sobre la base de las estimaciones de puntuación de factores. El porcentaje esperado de diferencias verdaderas (EPTD) es el estimado porcentaje de diferencias entre las estimaciones de puntuación de factores observadas que están en la misma dirección que correspondientes diferencias verdaderas. Si las 
puntuaciones de los factores se van a utilizar para la evaluación individual, valores de FDI superiores a 0,90, fiabilidades marginales superiores a 0,80, Se recomiendan SR por encima de 2 y EPTD por encima del $90 \%$ (Ferrando y Lorenzo-Seva, 2018). En la tabla 3 puede observar que todos, excepto factor 3, tienen FDI >0,90, fiabilidad $>0,80$, SR $>0,20$ y EPTD $>90 \%$ (Tabla 3). Sin embargo, el factor 3 es aceptable como un factor independiente.

El valor de Omega ordinal de McDonald's $=0,985$ y Alfa de Cronbach estandarizado $=0,985$. Donde el omega es el cuadrado de la correlación entre el puntaje de la escala y la variable latente común a todos los indicadores en el universo infinito de indicadores del cual los indicadores de escala son un subconjunto (McDonald, 1999).

Es importante destacar que los indicadores de Unidimensionalidad Congruencia Unidimensional (UniCo $=0,959)$, Varianza Común Explicada (ECV $=0,904)$ y Media de Cargas Absolutas Residuales del Ítem (MIREAL $=0,206$ ) apoyaron la unidimensionalidad de la escala. Un valor de UniCo (Congruencia unidimensional) e I-Unico (Congruencia unidimensional del elemento) mayor que 0,95 sugiere que los datos pueden tratarse como esencialmente unidimensionales.

Un valor de ECV (varianza común explicada) e I-ECV (varianza común explicada del elemento) mayor que 0,85 sugiere que los datos pueden tratarse como esencialmente unidimensionales. Un valor de MIREAL (media de las cargas absolutas residuales del elemento) e I-REAL (cargas absolutas residuales del elemento) inferior a 0.300 sugiere que los datos pueden tratarse como esencialmente unidimensionales (Ferrando y Lorenzo-Seva, 2018).

Tabla 4

Calidad y Efectividad de las estimaciones de puntuación de factor del Índice Global de la Educación Física de Calidad (GIQPE)

\begin{tabular}{|c|c|c|c|c|c|c|c|c|}
\hline & Factor 1 & Factor 2 & Factor 3 & Factor 4 & Factor 5 & Factor 6 & Factor 7 & Factor 8 \\
\hline $\begin{array}{c}\text { Índice de determinación } \\
\text { de factores (FDI) }\end{array}$ & .945 & .995 & .897 & .969 & .969 & .987 & .985 & .945 \\
\hline $\begin{array}{c}\text { Fiabilidad marginal de } \\
\text { ORION }\end{array}$ & .894 & .990 & .804 & .939 & .939 & .974 & .971 & .894 \\
\hline $\begin{array}{c}\text { Relación de sensibilidad } \\
\text { (SR) }\end{array}$ & 2.897 & 10.141 & 2.024 & 3.909 & 3.920 & 6.139 & 5.760 & 2.902 \\
\hline $\begin{array}{c}\text { Porcentaje esperado de } \\
\text { diferencias verdaderas } \\
\text { (EPTD) }\end{array}$ & $92.2 \%$ & $99.1 \%$ & $88.8 \%$ & $94.6 \%$ & $94.6 \%$ & $97.3 \%$ & $96.9 \%$ & $92.2 \%$ \\
\hline
\end{tabular}

\subsection{Discusión}

El objetivo de esta investigación fue analizar los parámetros psicométricos y las estructuras factoriales del Cuestionario de EFC en el contexto ecuatoriano.

Los tests de esfericidad de Bartlett $(p<.001)$ e KMO (.97) tienen una muy buena matriz de correlación como estructura interna. La mayoría de las preguntas del cuestionario presentaron cargas factoriales consistentes. Eso se corrobora con los índices de ajustes del instrumento $\left(x^{2}=628,63, \mathrm{gl}=853 ; p=0,999 ; \mathrm{RMSEA}=1,000\right.$; $\mathrm{CFI}=1,000 ; \mathrm{TLI}=1,000)$, y su medida de replicabilidad.

Hay una aceptable fiabilidad compuesta de los factores del cuestionario. Sin embargo, un fuerte valor de Omega ordinal de McDonald's $=0,985$ y Alfa de Cronbach estandarizado $=0,985$, como medidas de una buena estructura interna del instrumento en el contexto ecuatoriano. 
Al igual que sucedió con Rodríguez et al., (2021) al analizar la estructura factorial de este mismo cuestionario en México, los índices obtenidos son satisfactorios, con una consistencia interna de los factores excelente.

A través del cuestionario analizado se puede afirmar que ecuador goza de un instrumento que le permitirá conocer de primera mano la realidad de la EF a nivel nacional, hecho que le posibilitará la elaboración de políticas públicas que atiendan a las demandas de los profesionales del área de EF.

\section{Conclusiones}

Este tipo de investigaciones son sumamente necesarias, pues contribuyen a los retos que la sociedad tiene que enfrentar cada día, como en este caso, una Educación Física de calidad que permita, entre otras cosas, lograr la adherencia futura a la actividad física por parte de los alumnos.

Los resultados del presente estudio demuestran que los índices son satisfactorios, con homogeneidad entre los ítems de los factores. Por lo tanto, se puede afirmar que el Índice Global de la Educación Física de Calidad (GIQPE) es una herramienta que goza de las suficientes garantías psicométricas para ser aplicada en el contexto ecuatoriano.

Como principal limitación de esta investigación puede considerarse la manera en que se aplicó el cuestionario; pues la aplicación online puede ir acompañada de algunos inconvenientes, como la no posibilidad de resolver una duda "in situ" sobre una pregunta en concreto o el hecho de que la persona que responda el cuestionario no sea realmente un profesional del área de Educación Física.

\section{Referencias bibliográficas}

Asparouhov, T., y Muthen, B. (2010). Simple second order chi-square correction. Unpublished manuscript. Available at https://www.statmodel.com/download/WLSMV_new_chi21.pdf.

Brown, T. A. (2006). Confirmatory factor analysis for applied research. New York: The Guilford Press.

Contreras, O. R., Gil, P., Sebastiani, E., Pascual, C., Huguet, D., Hernández, J. L., y Capllonch, M. (2010). Didáctica de la educación física (Vol. 2). Ministerio de Educación de España. En:

https://sede.educacion.gob.es/publiventa/PdfServlet?pdf=VP13980.pdf\&area=E

del Val, P., Sebastiani, E., y Blázquez, D. (2021). ¿Qué es y cómo se mide la calidad en Educación Física? Una revisión de literatura. Sportis. Scientific Journal of School Sport, Physical Education and Psychomotricity, 7(2), 300-320.

del Val, P., Sebastiani, E., y Blázquez, D. (2021). Educación Física en Ecuador: análisis de la percepción de alumnos. Revista iberoamericana de psicología del ejercicio y el deporte, 15(4), 180-183.

Ferrando, P. J., y Lorenzo-Seva, U. (2018). Assessing the Quality and Appropriateness of Factor Solutions and Factor Score Estimates in Exploratory Item Factor Analysis. Educational and Psychological Measurement, 78(5), 762-780. https://doi.org/10.1177/0013164417719308

Ho, W., Dilsad, A., y Kukurova, K. (2021) Development and validation of an instrument to assess quality physical education. Cogent Education, 8:1, 1864082, DOI: 10.1080/2331186X.2020.1864082

Ho, W., Ahmed, D., Carvalho, P. G., Antala, B., Imre, M., Valeiro, M. G., ... Wong, B. (2019). Development of an instrument to assess perception of Quality Physical Education among the European professionals. South African Journal for Research in Sport, Physical Education and Recreation, 41(1), 31-49. 
Lara, L. M., Starepravo, F. A., Monteiro, A. C. de, y Matías de Souza, V. de F. (2018). Qualidade na Educação/Educação física escolar Latino-americana: encontro de vozes nada dissonantes. Journal of Physical Education, 29(1), e-2929. https://doi.org/10.4025/jphyseduc.v29i1.2929

López Pastor, V. M., Pérez, D., Manrique, J. C. y Monjas, R. (2016). Los retos de la Educación Física en el Siglo XXI. Retos, 29, 182- 187.

Lorenzo-Seva, U., y Ferrando, P.J. (2006). FACTOR: A computer program to fit the exploratory factor analysis model. Behavior Research Methods 38, 88-91 (2006). https://doi.org/10.3758/BF03192753

Lorenzo-Seva, U., y Ferrando, P.J. (2019). Robust Promin: a method for diagonally weighted factor rotation. Technical report, URV. Tarragona, Spain.

McDonald, R.P. (1999). Test theory: A unified treatment. Mahwah, NJ: Lawrence Erlbaum.

Pellicer, I. (2015). NeuroEF: la revolución de la educación física desde la neurociencia. INDE.

Pérez-Pueyo, A., Hortigüela-Alcalá, D., Fernández-Fernández, J., Gutiérrez-García, C., y Santos Rodríguez, L. (2020). Más horas sí, pero ¿cómo implantarlas sin perder el enfoque pedagógico de la Educación Física? Retos, (39), 345-353. https://doi.org/10.47197/retos.v0i39.80283

Reckase, M. D. (1985). The Difficulty of Test Items That Measure More Than One Ability. Applied Psychological Measurement, 9(4), 401-412. https://doi.org/10.1177/014662168500900409

Rodríguez, J., Ceballos, O., Zamarripa, J., Medina, R., Ho, W., y López, R. (2021). Educación Física de Calidad desde la perspectiva de la práctica docente: propiedades psicométricas de un instrumento para su evaluación (Quality Physical Education from the perspective of teaching practice: psychometric properties of an instrument for it. Retos, (41), 373-379. https://doi.org/10.47197/retos.v0i41.86253

Timmerman, M. E., y Lorenzo-Seva, U. (2011). Dimensionality assessment of ordered polytomous items with parallel analysis. Psychological methods, 16(2), 209-220. https://doi.org/10.1037/a0023353

UNESCO. (2005). UNESCO Seminar on Quality of Physical Education and Sport - Final Report.

UNESCO. (2015). Quality PE (QPE), Guidelines for Policy-Makers. Published in 2015 by the United Nations Educational, Scientific and Cultural Organization.

Vera Estrada, F.; Sánchez Rivas, E., y Sánchez Rodríguez, J. (2018). Promoción de la actividad física saludable en el recreo escolar. Revista Internacional de Medicina y Ciencias de la Actividad Física y el Deporte, 18(72), 655-668. En: DOI: http://doi.org/10.15366/rimcafd2018.72.004

Villafuerte, J., Pérez, L., y Delgado, V. (2019). Retos de la Educación Física, Deportes y Recreación en Ecuador: las competencias docentes (Challenges of Physical Education, Sports, and Recreation in Ecuador: the teaching competences). Retos, 36, 327-335. https://doi.org/10.47197/retos.v36i36.67062 